Lauren Sadow · Bert Peeters . Kerry Mullan Editors

Studies in

Ethnopragmatics,

Cultural Semantics,

and Intercultural

Communication

Minimal English (and Beyond)

Springer 
Studies in Ethnopragmatics, Cultural Semantics, and Intercultural Communication 
Lauren Sadow - Bert Peeters .

Kerry Mullan

Editors

Studies in Ethnopragmatics,

Cultural Semantics,

and Intercultural

Communication

Minimal English (and Beyond)

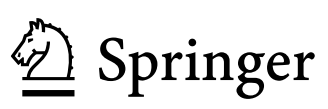




\section{Editors}

Lauren Sadow

Australian National University

Canberra, ACT, Australia

Kerry Mullan

RMIT University

Melbourne, VIC, Australia

\section{Bert Peeters}

Australian National University

Canberra, ACT, Australia

\footnotetext{
ISBN 978-981-32-9978-8

ISBN 978-981-32-9979-5 (eBook)

https://doi.org/10.1007/978-981-32-9979-5
}

(C) Springer Nature Singapore Pte Ltd. 2020

This work is subject to copyright. All rights are reserved by the Publisher, whether the whole or part of the material is concerned, specifically the rights of translation, reprinting, reuse of illustrations, recitation, broadcasting, reproduction on microfilms or in any other physical way, and transmission or information storage and retrieval, electronic adaptation, computer software, or by similar or dissimilar methodology now known or hereafter developed.

The use of general descriptive names, registered names, trademarks, service marks, etc. in this publication does not imply, even in the absence of a specific statement, that such names are exempt from the relevant protective laws and regulations and therefore free for general use.

The publisher, the authors and the editors are safe to assume that the advice and information in this book are believed to be true and accurate at the date of publication. Neither the publisher nor the authors or the editors give a warranty, expressed or implied, with respect to the material contained herein or for any errors or omissions that may have been made. The publisher remains neutral with regard to jurisdictional claims in published maps and institutional affiliations.

This Springer imprint is published by the registered company Springer Nature Singapore Pte Ltd. The registered company address is: 152 Beach Road, \#21-01/04 Gateway East, Singapore 189721, Singapore 
Dedicated to our good friend and colleague Cliff Goddard 


\section{Contents}

1 Minimal English: Taking NSM 'Out of the Lab' . . . . . . . . . . . 1 Lauren Sadow

\section{Part I Minimal English (and Beyond)}

2 Using NSM and "Minimal" Language for Intercultural

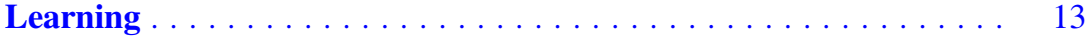

Susana S. Fernández

3 From Expensive English to Minimal English . . . . . . . . . . 33 Deborah Hill

4 "There is no Sex in the Soviet Union": From Sex to Seks . . . . . 53 Anna Wierzbicka and Anna Gladkova

5 When Value Words Cross Cultural Borders: English Tolerant Versus Russian Tolerantnyj. Anna Gladkova

6 The Confounding Mandarin Colour Term 'Qīng': Green, Blue, Black or All of the Above and More? . . . . . . . . . . . . . . . . . 95 Jiashu Tao and Jock Wong

7 Semantic Challenges in Understanding Global English: Hypothesis, Theory, and Proof in Singapore English . Jock Wong

8 Using Minimal English to Model a Parental Understanding of Autism

Alexander Forbes

9 Principles and Prototypes of a Cultural Dictionary of Australian English for Learners . . . . . . . . . . . . . . . . . . . . . 165 Lauren Sadow 
10 Minimal and Inverse Definitions: A Semi-experimental Proposal for Compiling a Spanish Dictionary with Semantic Primes and Molecules .

María Auxiliadora Barrios Rodríguez

11 Prevalence of NSM Primes in Easy-to-Read and Standard Finnish: Findings from Newspaper Text Corpora. . . . . . . . . 213 Ulla Vanhatalo and Camilla Lindholm

\section{Part II Cliff Goddard: List of Publications}

Cliff Goddard: List of Publications

Compiled by Bert Peeters 


\title{
Chapter 9 \\ Principles and Prototypes of a Cultural Dictionary of Australian English for Learners
}

\author{
Lauren Sadow
}

\begin{abstract}
This chapter discusses some of the issues that need to be considered when producing a user-friendly resource intended to familiarize ESL learners with the invisible culture of Australian English. It draws on specialized function lexicography (Tarp Lexicography in the borderland between knowledge and non-knowledge. Mouton De Gruyter, Berlin 2008) and on the cultural scripts approach as proposed by Goddard (Cognitive linguistics, second language acquisition, and foreign language teaching. Mouton de Gruyter, Berlin, pp 143-163, 2004). The resource takes the form of an encyclopedic dictionary focusing on Australian values, attitudes and interactional norms and aims to respond to an industry need for pedagogical materials that introduce migrants coming to Australia to the culture embodied in Australian English. Best practice for teaching cultural awareness and related skills is to use a method for teaching that encourages students to reflect on their experience and to analyse it from an insider or emic perspective (Pulverness and Tomlinson in Lang Teach 45(2):143-179, 2003). The cultural scripts approach, which deconstructs complex cultural elements into simpler and universally intelligible building blocks, provides an effective means to this end. The chapter contents that drawing connections between different cultural scripts and illustrating those connections in a way that promotes the acquisition of concepts for learners is one of the most important elements in cultural dictionary design.
\end{abstract}

Keywords Ethnopragmatics · Learner lexicography • English as a second language $\cdot$ Cultural awareness $\cdot$ Intercultural competence $\cdot$ Australian English

\subsection{Introduction}

Natural Semantic Metalanguage (NSM) scholars have more than once drawn attention to the relevance of cultural scripts theory and ethnopragmatics as potential resources for language teaching and learning in general, and for the teaching of

L. Sadow $(\bowtie)$

The Australian National University, Canberra, Australia

e-mail: lauren.sadow@anu.edu.au

(C) Springer Nature Singapore Pte Ltd. 2020

L. Sadow et al. (eds.), Studies in Ethnopragmatics, Cultural Semantics,

and Intercultural Communication, https://doi.org/10.1007/978-981-32-9979-5_9 
pragmatics and the acquisition of interactional competences, in particular (see e.g. Goddard and Wierzbicka 2004; Goddard 2004, 2010; Fernández 2016; Sadow 2018). In doing so, they have almost invariably focused on classroom pedagogies rather than learner resources. Goddard, in particular, has proposed a number of ways in which cultural scripts can be used to benefit students from different language backgrounds and has discussed, in several publications, how best to alter established cultural scripts for pedagogical purposes. In contrast, not much work has been done on how language learners can use cultural scripts theory and ethnopragmatics in their own private language learning. There is a clear need to develop student resources that make the best possible use of the pedagogical work that has been done to date, and that are primarily designed for independent study (but could still be used in the classroom as well). The format of a dictionary-i.e. a lexicographical format-appears to be the obvious choice for a pedagogical resource, focused on shared linguistic knowledge, that offers language learners access to emic perspectives on the languaculture they are studying. This chapter suggests that a combination of ethnopragmatics and lexicography can benefit language learners in a way that furthers their acquisition of interactional, strategic, cultural and intercultural competences.

The combination of ethnopragmatics and lexicography is innovative. ${ }^{1}$ It is one of the hallmarks, if not the most outstanding feature, of my ethnopragmatic dictionary for learners of English, the Cultural Dictionary of Australian English for Learners (CDAEL), which is the focus of this chapter. The idea of a learners' dictionary containing cultural content is a direct challenge of lexicographical convention, since the standard expectations of dictionary content are clearly not adhered to, particularly in terms of headwords to be defined. The CDAEL uses so-called compositions to articulate ethnopragmatic information (as well as some culturally salient lexical information) and convey it to the audience of English language learners from diverse backgrounds. Compositions are written in Minimal English (Goddard 2018), a by-product of NSM that provides a flexible framework for developing pedagogically oriented cultural scripts and explications in a standardized format. The term 'composition' aims to capture the careful and methodical nature of explications and cultural scripts written in Minimal English. It can be used to summarize a combination of explications and cultural scripts, or to describe a piece in Minimal English that does not naturally fall into either category. By using Minimal English, the information is made accessible to all learners, without losing its cultural significance.

While the CDAEL is still under development, some prototyping has already taken place (cf. Sadow 2019). The Australian Dictionary of Invisible Culture for Teachers (AusDICT) (Sadow 2019) is based on the same principles, but is aimed at English language teachers, rather than language learners, as its main users. This version of a Minimal English-based dictionary of ethnopragmatics used design-based research (Amiel and Reeves 2008) to consult with language teachers

\footnotetext{
${ }^{1}$ Publications that connect NSM and lexicography have typically focused on lexical semantics and have not included pragmatics.
} 
around Australia to develop the content, structure, and general format of the book. Many of the principles emerging from the design-based research are discussed throughout this chapter. However, it will be useful to briefly illustrate the prototype.

The AusDICT includes both ethnopragmatic entries, as well as entries for cultural keywords (Levisen and Waters 2017), as both were articulated by teachers as needing further resources for teaching. Figure 9.1 illustrates the AusDICT entry for whinge, a cultural keyword of Australian English (Wierzbicka 1997).

Figure 9.2 illustrates the AusDICT entry for 'doing something when something bad happens', a widespread way of thinking among speakers of Australian English (Wierzbicka 2002).

These two examples show how a more Minimal English approach to writing ethnopragmatic scripts for pedagogical purposes can make the information contained therein more practical for pedagogical contexts. They also show that the principles of lexicography and dictionary-making, such as including examples, structuring entries to draw attention to particular aspects, and indicating related terms, and support the more non-traditional entries.

As previously indicated, AusDICT is aimed at English language teachers. The target users of the CDAEL (the focus of the current chapter) are language learners. There are specific knowledge, pedagogical and user needs unique to this target

whinge " $X$ was whingeing"

verb: someone does something

For some time, someone (e.g. Ken) was saying something like this "Something bad is happening to me."

Ken was saying it like people say things when they want to say something like this "Something bad is happening to me. I feel something bad because of this. I can't do anything. I want someone to know this. I want someone to do something because of this. I think no one wants to do anything. I want to say this many times because of this."

People think it is bad if someone does this often.

"I know I whinge too much. Thanks for listening to me."

"Val, stop whingeing. You need to come up with a plan and just do it."

doing something when something bad happens tough attitude anti-whingeing whinger sook

Fig. 9.1 Entry for whinge from the AusDICT (Sadow 2019) 
doing something when something bad happens

attitude: some people can think like this about something

When a person feels something bad, it is good if this person

thinks: I want to do something because of this.

When someone thinks like this, it is good if they do something because of this.

"Sasha's car has broken down. Sasha feels bad because of this. Sasha thinks "I can do something now. I can fix some things. If I can't fix it, I can call someone for help." Because of this, Sasha looks at the problem, and then phones for some help."

"Alright then. They don't have any small boxes in stock in any of their stores, and I need it tomorrow. I will get the things to make them instead. It's more work, but I will have them like I need."

she'll be right tough attitude anti-whingeing battler digger whinge

Fig. 9.2 Entry for doing something when something bad happens from the AusDICT (Sadow 2019)

audience. In this chapter, I engage with the three key fields that need to be united for the CDAEL project to be viable. The first of these is second language teaching and learning, more specifically the creation of language education materials. The second is lexicography, in particular learner lexicography. The third is ethnopragmatics. I discuss the contribution ethnopragmatics can make to second language teaching and learning, materials creation and learner lexicography, and I conclude with a discussion of the practicalities of creating an ethnopragmatic dictionary for learners.

\subsection{Ethnopragmatics for Teaching}

\subsubsection{Ethnopragmatics for Teaching What?-Knowledge Needs}

The first thing to be clear about is what ethnopragmatics should be used to teach, and why. Ethnopragmatic cultural scripts do not define the semantic content of lexical items in the same way that NSM explications do. Instead, they capture detailed and emic perspectives on the ways in which speakers interact, and the values and attitudes that underpin these behaviours. As a result, their titles are all 
rough 'translations' or recordings (usually in English) of the deconstructed concept. In some cases, this is a concept with terms attached (as in 'jocular abuse'; see Goddard 2017a), sometimes it is epitomized by an expression (as in 'you don't abandon a mate'; Wierzbicka 2002), sometimes it is a concept best expressed through a number of explanatory words (as in 'it's my house, I do what I want'; Wierzbicka 2012). The examples in [A] and [B] illustrate the difference between an NSM explication and a cultural script (which reflects a way of thinking, rather than the semantic meaning).

[A] X was whingeing (Wierzbicka 1997: 215)

for some time, $\mathrm{X}$ was saying something like this:

something bad is happening to me

$X$ was saying it as people say things when they want to say something like this:

something bad is happening to me

I feel something bad because of this

I can not do anything

I want someone to know this

I want someone to do something because of this

I think no one wants to do anything

I want to say this many times because of this

people think: it is bad if someone does this

[B] A cultural script for 'doing something when something bad happens' (Wierzbicka 2002: 1198)

when a person feels something bad, it is good if this person thinks:

'I want to do something because of this'

Each of the concepts articulated in ethnopragmatics map onto 'culture' of some description, but within the overlap between pragmatics, sociopragmatics, and culture. In many ways, each of those linguistic elements has a culturally mediated component that has been described using cultural scripts. In language teaching, each of these aspects of language map onto a different competence: pragmatic, strategic, sociopragmatic, and cultural. These competences comprise what is generally accepted as the ideal aim for language learners to achieve-intercultural competence (IC) (Byram 1997; Kramsch 1993; Liddicoat and Scarino 2013). IC has many different aspects, but is underpinned by an understanding of how cultures and people differ on the level of values and interaction, and strategies to navigate interactions outside of one's own cultural background. Also important is the ability to value other cultures in the same way as one's own, and to be able to analyse and develop understandings about situations in real time.

These competences are essential for language learners because language use is heavily linked to identity and self-expression (Liddicoat 1997). Without the tools to express and manage their image in their second language, learners find it difficult to 
be accepted into a community, to make meaningful relationships, or even to be understood, which leaves them susceptible to otherwise avoidable mental health challenges (Department of Social Services 2014). Journal articles, books and newspaper interviews illustrate again and again how isolation is among the most significant struggles faced by migrants and international students, especially from non-native English speaking backgrounds (e.g. Huang 2016; Mills 2018). Importantly, it is clear that the implicit instruction received by language students is not sufficient to address their problem, and particular care should be taken to provide explicit instruction in pragmatics, sociopragmatics and culture in classroom contexts and materials targeted at language learners.

That culture has a significant role in language teaching and is now the prevalent position of language educators and researchers (Risager 2006). At first glance, it appears that there are numerous resources for language learners to learn cultural content. However, upon closer inspection, the suggested methods are incomplete both in scope and in appropriateness of the materials to students of diverse backgrounds. Ethnopragmatics can intervene here and demystify the values, assumptions, and interactional norms experienced by native speakers of the language. By describing each of these in cross-translatable terms and breaking them down into a series of components that capture how native speakers conceptualize the world, ethnopragmatics can fill an apparent gap in the education of language learners.

\subsubsection{Ethnopragmatics for Teaching How?_Pedagogical Needs}

The theory for creating language teaching materials has specific recommendations for materials aimed at cultural instruction (Pulverness and Tomlinson 2003), as well as pragmatics and other associated skills. The recommendations stem from Pulverness and Tomlinson's observation that teaching materials do not explicitly address culture in nuanced ways, focusing more on the visible elements, rather than critically engaging with cultural diversity and emic perspectives. Ethnopragmatics inherently provides the emic perspectives (Goddard and Ye 2015), and through the structure of compositions is also able to provide nuance between different approaches to interaction and individual variation. Teaching materials can be considered to be anything a student uses to promote learning (Tomlinson 2012), including material in and outside of classrooms, as well as pedagogically directed materials (such as homework, textbooks, and assigned reading .) or self-directed materials (such as YouTube videos, TV shows, podcasts, newspapers, and self-chosen novels). These categories overlap in many ways. The intention is to acknowledge the role that all materials play in the teaching of language, not just traditional classroom worksheets. Importantly, this definition of teaching materials includes dictionaries. 
Materials for teaching culture should promote the same kinds of skills and attributes expected of an interculturally competent speaker. They should encourage students to:

- Accept that one's practices are influenced by the cultures in which one participates, and so are those of one's interlocutors;

- Accept that there is no one right way to do things;

- Value one's own culture and other cultures;

- Use language to explore culture;

- Find personal ways of engaging in intercultural interaction;

- Use one's existing knowledge of cultures as a resource for learning about new cultures;

- Find a personal intercultural style and identity. (Liddicoat and Scarino 2013: 23-24)

To achieve this, materials need to be able to be targeted at developing these attributes. Many of these are not just about the L2 culture, but about recognizing cultures in contrast to one another, and recognizing the ways in which individuals within those cultures can vary while not promoting any as being superior to the others.

Keeping this in mind, it is interesting to note that individual and sub-group variation is a nuance often missed in cultural teaching materials (Weninger and Kiss 2013). For the most part, 'cultural' materials present a monolithic view of culture, facts, figures, and enhance stereotypes (e.g. Usó-Juan 2008). However, the real need is for students to engage critically and reflectively with materials, but also to be able to understand the underlying reasons and causes of cultural differences. Cohen and Ishihara (2012) and Pulverness and Tomlinson (2003) discuss the ideal qualities of teaching and learning materials for pragmatic and cultural awareness; these can be summarized as follows:

- Materials should use real speech (or text) examples;

- Materials should give explicit information on situational and contextual variation (including gender usage);

- Materials should explain the cultural reasoning for norms;

- Materials should connect pragmatic and interactional information to vocabulary;

- Materials should provide information to teachers and learners on norms and pragmatics;

- Materials should encourage student to develop analytic skills so they can become ethnographic observers.

A key theme emerging in these suggested qualities of good teaching materials is that the information on culturally mediated features of language should be explicit in teaching materials and given time for learners to focus on.

Naturally, many of these qualities are far easier to capture in classroom tasks than in reference material. However, the cultural scripts approach to describing cultural norms and pragmatics captures much of this information as explicitly and 
clearly as is possible. It provides situational context, permits for variation in differing degrees, explains the cultural reasonings behind norms, and can be connected to vocabulary. In addition, by breaking down the pragmatic and cultural concepts into components, cultural scripts encourage readers (and therefore learners) to think about each part on its own, thus developing their analytic skills in interactions.

The challenge for a dictionary containing this information is to present it in a way that is accessible for language learners, so that they are able to retrieve as much of that content from within the cultural scripts as possible.

\subsection{Learner Lexicography and User Needs}

A dictionary is designed based on the functions it should perform for its users in order to meet their specific needs (Tarp 2008). The CDAEL is not intended to be used exclusively in classrooms with teacher support, but rather for it to be reference material that is accessible in the day-to-day lives of language learners. This means that the material needs to stand on its own, and be accessible to non-native speakers, despite being a monolingual dictionary.

Learner dictionaries vary based firstly on whether they are providing encoding or decoding information to users. Encoding dictionaries assist users to find the best way of expressing their intended message, while decoding is an interpretive task, helping users to understand native speaker utterances. An encoding dictionary of ethnopragmatics could be seen as prescriptive, implying that non-native speakers must conform to native speaker standards - an idea now disfavoured in language pedagogy research (Kramsch 2014). The CDAEL focuses instead on decoding as its main function by framing cultural scripts from a hearer's perspective (e.g. 'when someone says to me...') rather than the speaker's perspective (e.g. 'when I want to say...').

Still under debate is where to draw the line on what information constitutes a lexicographical work. While some lexicographers argue there ought to be no difference between dictionaries and encyclopedias, many argue that the distinction is required (Peeters 2000). At the heart of this debate is the issue of which information should be considered encyclopedic and which semantic. Goddard (2011: 16) draws a distinction between the two by saying that 'linguistic knowledge is essentially shared between all the speakers of a language, whereas real-world knowledge is not'. Ethnopragmatics, being directly related to language as shared by the broad speech community (in awareness if not in practice), comprises linguistic knowledge, and not encyclopedic knowledge. Building on this concept of linguistic knowledge is the developing field of cognitive lexicography, which advocates for the inclusion of cognitive information alongside the semantic, as well as for the better design of dictionaries in line with our cognitive conceptualizations of concepts, rather than mere alphabetical order (Ostermann 2015).

The needs of dictionary users have changed significantly in the last twenty years - most importantly because of the prevalence of the Internet and technology today 
(Lew and de Schryver 2014). As a result, print dictionaries are selling fewer copies, but online access is popular. This of course presents another challenge for lexicography as an industry, but this question will not be discussed here. From a user perspective, however, the convenience of something as portable as online access (or a smartphone application) is significantly preferable to a paper-based resource. As a result, the ideal learners' dictionary in this case is electronic and searchable.

\subsubsection{Dictionaries as Educational Tools}

Learner dictionaries in educational contexts have an essential role in language teaching and learning as they provide definitive, reliable reference materials for both students and teachers on the meanings and usages of words. At the same time, they help students to develop skills in independent research, analysis and critical thought about language use. In this way, dictionaries are used as teaching materials in classroom exercises, but they also feature in student learning outside the classroom as independent skills development. Other materials can perform similar functions, but none has the breadth of information across all topics that a dictionary does.

Using dictionaries as teaching materials can also have its drawbacks. Most dictionaries do not contain critical discussion of the words and phrases defined within them (even if they can be used to start that critical discussion), and they are so packed with information that it can be difficult to discern related topics and boundaries of material. This leaves the development of such questions and the generation of these discussions up to teachers, who may not have the knowledge or confidence in these topics to discuss them in an in-depth manner.

A dictionary designed for the teaching context could be arranged with conceptual domains or teaching topics that would guide teachers and students through the material. Such a design would enhance the connectedness of the ideas discussed, without sacrificing the accessibility gained through alphabetization. Some of the issues related to structuring an ethnopragmatic dictionary in this context are discussed in Sect. 9.6.

\subsubsection{Learner Dictionaries}

Although the first learner dictionaries were created in the 1930s, there is still no coherent methodology for creating them (Tarp 2008). I suggest this is in part because they are approached as purely lexicographical exercises, rather than language teaching materials. Fuertes-Olivera (2010) suggests that, to remedy some of the problems with learner dictionaries, companies ought to cater to a specific user with an explicit language ability and use purpose instead of catering to the broadest possible range of users. In addition, learners need to acquire language skills in 
addition to language knowledge. However, 'skill' is not a category that is incorporated into lexicographical theory (Tarp 2008). Some innovations and additions in recent years have tried to rectify this omission (see below). Nevertheless, this content has been made supplementary to the original definitions-creating the impression that the meaning and the cultural connotations associated with it are separate parts of the language and are not intertwined (Sánchez 2010).

That said, learner lexicography has been marked by some interesting innovations compared to traditional lexicography. The Longman dictionary series, in particular, has made innovative decisions regarding flow and organization. In the Longman Essential Activator (LEA) (Pearson Education, Inc. 2006), articles are organized by theses and related terms, not alphabetically. The LEA is designed for encoding purposes: it allows users to explore connected ideas through a single word, thereby enabling them to find the word that best suits their expressive needs, rather than the word that most literally translates a word in their mother tongue.

A problem mentioned by both Tarp (2008) and Fuertes-Olivera (2010) is the difficulty in selecting headwords for learners' dictionaries. Both state, it is impossible to include every item in a dictionary aimed at learners, but the process for deciding what to include is unclear-how much should be included, and what is of greatest importance to the users. Currently, there is no methodology for determining which headwords belong in dictionaries, regardless of whether they are aimed at native speakers or at learners. Seargeant (2011) points out that the status of English as a global language adds to the difficulty. The proposal of this chapter is that the required resource be specific to one particular variety of English (or that each variety requires its own resource), not generalized across all Englishes. While there is some overlap in interactional styles between varieties of English, it is also important to have resources with the different varieties as focal points and this does raise questions of granularity-how specific is that variety of English? If 'Australian English' is the variety chosen, how is that variety subsequently defined? What happens to varieties such as Aboriginal Australian English? Should regional variation be included beyond slang? How are those regions delineated? Etcetera.

In selecting headwords for this dictionary, there is an advantage in the specificity of an ethnopragmatic dictionary. The headwords need to be relevant to ethnopragmatics. However, bearing in mind the tenets of good design of teaching materials, they should also be linked to real language use and real phrases. Therefore, some lexical headwords (such as cultural keywords, see Fig. 9.1) are included in addition to ethnopragmatic headwords (e.g. Fig. 9.2). In terms of scope, the extent of ethnopragmatics that can be described is enormous. It is important to narrow this down to resolve issues of information density mentioned above. This can be done through relating words to classroom teaching topics, e.g. the 'units of instruction' offered by ACARA (2011) or the AMEP (Department of Education and Training 2011). It can also be done through the levels of cultural scripts offered within the field of ethnopragmatics. Perhaps it is best to include only master-level scripts, or only interactional scripts, as the remainder are not relevant to student experiences. This will be discussed further in Sect. 9.5. 


\subsubsection{Learner Needs and Teacher Needs}

User research on a proposed ethnopragmatic dictionary shows that the needs of both language learners and teachers need to be considered in creating materials for classroom practice. Both are important, because even materials used exclusively by learners need to be integrated into the class content, or otherwise fit into the curriculum. At the same time, the dictionary cannot rely on that context, as its intended audience is not exclusively located in classrooms.

Trials of the NSM framework with English language students have shown that the approach is suitable for teaching contexts (see Sadow 2018). However, for a dictionary requiring students to access the data by themselves, the use of Minimal English rather than NSM was preferred by those students. Additional adjustments to Minimal English are discussed in Sect. 9.5.1.

Teacher needs, on the other hand, were both more general and more explicit. Through a teacher survey and several focus groups, language teachers elaborated on the content they needed to teach, as well as the pedagogical requirements of resources aimed at language students. In summary, from their perspective, an ethnopragmatic dictionary needed to be:

- Contextualized - i.e. able to be related to the students' lives, particularly their needs and questions about language and culture, as well as the content being taught in their classes;

- Practical-i.e. providing practical direction on how to implement the ethnopragmatics in interactional contexts, rather than constrained to a theoretical discussion of values;

- Accessible - i.e. teaching and learning resources need to be portable and accessible for their students, meaning that an online dictionary, or a smart phone application is the ideal publication method for an ethnopragmatic dictionary, not a paper publication.

The main concern for all teachers in the focus groups was that the material be of maximum applicability to their students, and that their students be able to develop effective communication skills by using such a resource.

\subsection{Lexicography in Practice}

\subsubsection{Digital Versus Paper Dictionaries}

In the current age of lexicography, electronic lexicography (e-lexicography) dominates the field. Even in the creation of paper dictionaries, electronic databases and coding systems are the norms in maintaining the large number of entries and articles of commercial dictionaries. In turn, online dictionaries use similar systems to code and store their information. E-lexicography is a currently exploding field, as computer technologies develop capabilities across domains and become more 
accessible to people globally. As discussed above, an ethnopragmatic dictionary for learners is most practical if developed as an online dictionary.

Online dictionaries are generally accessible through websites but also smartphone applications. They usually have a search function to help users find the terms they are looking for, making them ideal for information seeking. The search terms are not obliged to be the same as the headwords of an entry, and several search terms can lead to the same point (Tarp 2008).

Also, importantly, online dictionaries do not have visible alphabetical structures. The entries in their database are stored and categorized in several different ways, which means that users can access the information in the same kinds of ways. This may be through the multiple search terms mentioned above, or via explore functions, designed by different types of domains, or even via common components in entries. The Longman Essential Activator (2010) presents a paper version of how this could be achieved. In an online dictionary, however, it is much easier to have headwords and entries belonging to several different domains, bridging domains, etc. This further improves accessibility and discoverability of connected and useful information.

The key to creating online dictionaries is in creating effective coding systems for a database in a markup language that can be used across multiple platforms and in different iterations. For the proposed dictionary, I am using XML to mark up the entries to be maximally useful across platforms and projects-now as well as into future.

\subsubsection{A 'Good' Definition}

There are three key principles in writing good dictionary definitions (Landau 2001). First, a definition should not be circular: it should not refer back to itself either in the first instance or in subsequent definitions of included words. Second, definitions should not include words more complex than the word being defined (Zgusta 1971) and every word used in a definition should be defined in the dictionary. Third, items must be sufficiently defined, i.e. definitions must suit the dictionary's function and should neither capture only encyclopedic information nor be too limited in its semantic information. These principles are remarkably close to the key principles of good NSM explications. However, NSM also requires definitions to be cross-translatable.

Considering that the target user of the proposed dictionary is a language learner, using cross-translatable language to capture culture-specific concepts ensures users from all backgrounds can access the information in the same way. This also widens the user base of the dictionary, rather than constraining it to a single L1 user group.

It is a common practice in lexicography to ensure definitions are as short as possible - space in paper dictionaries is at a premium (Landau 2001). Concision 
means commercial dictionaries can accommodate more entries. This is of core importance to commercial publishers, as the number of entries is a major criterion for judging lexicographical works (Atkins and Rundell 2008).

\subsubsection{NSM/Minimal English as a Defining Vocabulary}

Many learner dictionaries use a limited defining vocabulary (Atkins and Rundell 2008). Defining vocabularies improve the ability for non-native speakers to understand a monolingual dictionary by using a limited set of words. Most are made up of 1000-2000 words, with some flexibility for derived forms - significantly more than the number allowed in NSM, which lexicographers often dismiss as a defining methodology in part because of lexicographical conventions and established expectations in the market, but also because of space and time constraints (Atkins and Rundell 2008). The choice of words is usually based on frequency in the language, not on principles of translatability, which means that many are highly culture specific. Words such as reasonable and sense (Wierzbicka 2010) are often included. In contrast, NSM provides the basis for a highly controlled vocabulary that increases definitional accuracy. Not only are NSM definitions more accurate because they are more detailed; their accuracy is also enhanced by the fact that they are able to include the associations and scenarios that exist in a language user's understanding of a concept, rather than just the semantic information. NSM definitions have the added benefit of being cross-translatable across a wide range of languages and language families and more accessible for non-native speakers. NSM also provides a defining syntax for language learners, not only a vocabulary. As a result, readers are guaranteed a certain degree of consistency between the entries, as well as the certainty that the meaning is consistent with their translation of the meaning. This is especially important for lower levels of language learners.

The NSM approach includes both semantic primes and semantic molecules. It makes sense in a lexicographical context to also use semantic molecules to explain complex concepts (and in some cases, this is essential). However, as semantic primes are considered the core units of meaning in any language, they are as such indefinable in any simpler terms. This means that it is not possible to define them within a dictionary based on NSM principles. Previous NSM-based dictionary projects (see e.g. Bullock 2011) have resolved this challenge by first defining each prime pictorially before using them in definitions. I propose, however, that this learner dictionary does not need to do this, as the concepts ought to be well established for users of the dictionary. It would perhaps be possible to provide the list of primes in a number of languages to avoid complete opacity in meaning. Lists of primes in over 20 different languages are currently available on the NSM homepage at Griffith University, Australia. 


\subsubsection{Key Features in Entries}

While the definition is one of the key parts of a dictionary entry, it is not the only part of an entry that provides information to the user. In fact, a good dictionary will encourage its users to develop a greater understanding of the entries than their query required (Landau 2001). In creating a new dictionary not based on previous lexicographical publications, each of the features of an entry should be considered and their realizations should be reconsidered so that they meet the users' specific needs within the function of the dictionary. I will consider some of the key features expected in dictionaries here and will discuss their use and realization in the proposed ethnopragmatic dictionary.

\subsubsection{Headwords}

The first component of an entry that users encounter is the headword. For most users, this is how they access information in a dictionary. Normally, the headword is a lexical item and the presentation of the headword also gives standard spelling/s. In the case of ethnopragmatic entries, the specific headword functions only as the access point for the entry. However, as discussed below, this has some challenges for the types of headwords required for cultural scripts versus explications.

\subsubsection{Pronunciation}

Headwords are traditionally followed by a pronunciation of some sort, which is particularly important for learner dictionaries. Many dictionaries provide this pronunciation guide in the International Phonetic Alphabet (IPA), but some dictionaries use a 'literal spelling' guide, assuming that their users do not have familiarity with the IPA. In the case of ethnopragmatic entries, it is not possible to give pronunciation guides for values and attitudes. However, where entries represent specific words or phrases, IPA can give a guide to how these are said. It would be more useful to develop a way of capturing tone of voice and other extralinguistic features of speech that influence how these utterances are to be interpreted.

\subsubsection{Part of Speech}

The part of speech of the entry is important information governing the grammatical usage of the entry, and the context of the definition. Again, this is only relevant where the entries are lexical or phrasal in nature. Explications traditionally begin with a phrase that indicates the lexico-syntactic frame of the term being explicated (Goddard 2011) and that serves the same purpose as the part of speech label. As this is a learner dictionary, however, I propose that both these elements be combined to 
provide additional information to the user on the frame being explicated, but also on how it fits into their traditional model of language learning.

\subsubsection{Usage Labels}

Usage labels occur in different forms and provide information about different types of usage - typically with the help of specific 'codes'. A common usage label is that of regional usage. While many learner dictionaries cover several language varieties, the proposed dictionary only covers Australian English; specifying country of origin is therefore unnecessary. This kind of nation-based specification is habitually the only type of regional variation found in dictionaries. However, other types of regional variation exist within Australian English (Moore 2008) and these regional differences (such as state/rural/urban) would be useful for language learners.

Other kinds of usage labels, such as register, also contain useful information for language learners, but in the case of the proposed dictionary are not needed as the information is already contained within the Minimal English compositions in phrases such as 'it can be bad if I say this to someone above me' or 'people feel something very bad when they hear this word'.

\subsubsection{Notes}

A common feature in learner dictionaries is the additional usage information (or sometimes encyclopedic information) in additional notes appended to entries. These notes are generally short in paper dictionaries and can be longer in digital dictionaries, but often only elaborate on a single point of usage - usually historical or social context. Much of this information is also included in the Minimal English compositions that comprise the definitions. On occasion, these notes also point out certain potential pitfalls for learners, such as other items the term should not be confused with, and points of difference between this and another entry. This kind of note would be particularly useful for language learners especially considering the different kind of definitions they are encountering. In addition, these notes would be an ideal place to highlight certain key points of the composition, such as particular usage of 'bad' vs. 'very bad' or where the 'can' is particularly relevant. In an online dictionary, these notes can be appended to every entry, thus providing users with key pieces of information at every opportunity.

\subsubsection{Cross-references}

Finally, and potentially most crucially, cross-references can maintain the complex connections between cultural values and present these connections in ways users can learn from. Some traditional dictionaries use cross-referencing sparingly, in notes such as those discussed above. In most cases, cross-referencing occurs where 
there are two words for a concept and searching the less common headword will cross-reference to the more common one, where the entry is. In an ethnopragmatic dictionary, using cross-referencing to connect cultural values to attitudes, interactional norms and utterances is an important way of teaching language learners about how invisible culture influences the ways in which native speakers interact. In an online dictionary, extensive use of hyperlinking and mouse-over features makes drawing these connections easier, resulting in the ability to include more of them, more often, on more entries.

\subsection{A Minimal English for Learner Lexicography}

As is to be expected, the NSM approach requires some adaptations for use in pedagogical and lexicographical contexts. Goddard (2017b) presents some explications in a lexicographical format that, in essence, has no line breaks and is a condensed form of the explication (in form, not in content). However, building on the research with learners mentioned above, I would suggest there are other adaptations that would be beneficial in a learner's lexicography context. They are meant to improve readability, retrievability, and relatability for users. In many ways, they bring the cultural scripts towards Minimal English. However, this is a variation of Minimal English for a very specific purpose. As described above, there is more to a dictionary entry than the definition itself. Each of these parts should be carefully considered for a dictionary of ethnopragmatics, as well as being considered alongside the recommendations for good materials for developing intercultural competence.

If we compare the semantic explication in [A] to the AusDICT entry for whinge in Fig. 9.1 (both repeated here for convenience), the changes from NSM to a Minimal-English-like phraseology are apparent in both wording and formatting.

[A] X was whingeing (Wierzbicka 1997: 215)

for some time, $X$ was saying something like this:

something bad is happening to me

$X$ was saying it as people say things when they want to say something like this:

something bad is happening to me

I feel something bad because of this

I can't do anything

I want someone to know this

I want someone to do something because of this

I think no one wants to do anything

I want to say this many times because of this

people think: it is bad if someone does this 
whinge "X was whingeing"

verb: someone does something

For some time, someone (e.g. Ken) was saying something like this "Something bad is happening to me."

Ken was saying it like people say things when they want to say something like this "Something bad is happening to me. I feel something bad because of this. I can't do anything. I want someone to know this. I want someone to do something because of this. I think no one wants to do anything. I want to say this many times because of this."

People think it is bad if someone does this often.

"I know I whinge too much. Thanks for listening to me."

"Val, stop whingeing. You need to come up with a plan and just do it."

\section{doing something when something bad happens tough attitude} anti-whingeing whinger sook

Fig. 9.1 (repeated). Entry for whinge from the AusDICT (Sadow 2019)

\subsubsection{Language Adaptations}

As described in Sect. 9.3.3, engaging with user needs is essential to the successful creation of a dictionary, especially in such a new paradigm. The added challenge comes in using Minimal English as a defining vocabulary and syntax. Therefore, Minimal English too should not escape examination by the potential users of the resource. From this research, users made some recommendations that are also discussed here.

The first of these recommendations validate the need for Minimal English in place of NSM as a defining language. The potential users felt that the restrictions of only 65 concepts were too limiting to clearly define the terms they needed. For the teachers, some of the additional words needed were relevant specifically to classroom contexts, such as 'student' 'teacher' 'class' and 'classroom'. Other words helped to simplify more complex concepts (as all molecules do in Minimal English). As for the students, they often felt that their vocabulary was larger than the 65 words and therefore they would be able to manage a larger defining vocabulary. Most of the students were in intermediate-advanced classes, so while this may be true of these students, it may not be true of others.

The second of these recommendations was to simplify the repetition in the Minimal English compositions. In particular, students found the repetition of 
'someone' 'this someone' 'this other someone' 'another someone' to be confusing. As a result, they preferred either 'person A' 'someone (A)' or even using names as examples such as 'someone (e.g. Mark)'. The challenge with using names in compositions is that it can unintentionally give the impression that the compositions are gendered in one way or another. In a dictionary, this can partly be resolved by either using gender neutral names, or by specifying with lines such as 'someone can say something like this if this someone is a man'. This is not dissimilar to the pedagogical suggestions in Goddard (2010), who notes that additional components may be required for learners from different backgrounds. Despite this challenge with using names in compositions, the comments from teachers pointing out the need to make the compositions as relevant as possible to the lives of students mean that names are one of the most explicit ways to do this.

Goddard (2017b) uses examples that do not exceed a single paragraph of definition in the NSM metalanguage. These definitions are precise, but also generalized. However, the longer the definition, the more difficult it can be for a reader to build up a complete picture of the meaning. This is especially important for ethnopragmatic compositions, as the different components are each important for the interaction. Losing a single piece of information can influence the reader's understanding. In fact, the language learners liked the presentation of the explications, saying that the 'step-by-step' approach was very clear to them (Sadow 2018). However, the teachers noted that many of the line breaks in place of subordinate clauses and full stops were difficult for them to understand. Given this, I propose that a simplified version of the NSM explication structure be adopted, as illustrated in Fig. 9.1. In this version of Minimal English, some of the line breaks used in NSM are retained, but where indented lines would indicate subordinate clauses, they have been included as sentences in the same paragraph. This helps to keep connected ideas together, but it also keeps the flow of the compositions.

\subsubsection{Headwords}

One of the most complex considerations to make is the actual headword itself. This is a complex issue because it determines how the dictionary user accesses the information. However, in the NSM methodology, the cultural script itself should stand on its own without the need for a 'summary in a single word or phrase' provided by a headword. In some NSM publications, no title to an explication is given, and in some the title is a rough description in more complex language of what the cultural script is determining. These titles provide a reasonable starting point for the 'headword' of an entry. Although the challenge is determining how these headwords should be articulated. As discussed in Sect. 9.4.1, e-lexicography provides some answers to this challenge, through the use of 'invisible' headwords, and through attaching multiple headwords to the same entry (Tarp 2008). Combining both, it is possible for a user to then find the most relevant entry by searching several terms and being provided with the entry and composition without 
a headword. Despite this as a possibility, it is more effective for users to have a single visible headword so that they can assess relevance when presented with several alternatives.

In the CDAEL, headwords that are reminiscent of the titles of cultural scripts are used because they express some of the complex ideas in plain language. The example below, based on Goddard (2012: 116), shows the 'headword' for a cultural script for projecting presumed solidarity in interaction.

Projecting presumed solidarity in interaction

Many people in Australia think like this:

When I say something to someone, it is good if they can know that I think about them like this:

This person is someone like me.

Other possible headwords that could function as 'invisible' headwords in addition to the visible one include 'showing presumed solidarity in conversation', 'demonstrating solidarity', 'showing you are similar to someone', and so on.

\subsubsection{Examples}

Many dictionaries contain examples, often from the media or constructed to illustrate certain meanings. For an ethnopragmatic dictionary, including examples of real speech is essential to capture contexts of the cultural scripts. Because many of the cultural scripts are not necessarily connected to specific words or phrases, several examples where the interactional norm or the value is evident in a conversational routine is ideal. Examples such as these are almost impossible to include in a paper dictionary, as they would take up too much space. However, in an online dictionary, space is of no concern, but more importantly, it is possible to include such examples as video or audio interactions, as well as text-based material.

\subsubsection{Phrases/Connection to Language Production}

As discussed in Sect. 9.2.2, it is important when teaching ethnopragmatic skills to include information about phrases and language production. Therefore, the dictionary should also, where relevant, include reference to phrases and utterances. In most cases, there is a large range of phrases that can capture that interactional intention, or else it can be captured in tone of voice alone. However, some indication is still needed for language learners, as long as there is acknowledgement of additional possibilities. Inclusion of this element could be done in several ways. 
First, through the examples discussed above. In many cases, the examples of interaction will have to include linguistic components. With several of these, it would be possible to capture different realizations of the interactional norm. Secondly, they could be included through additional notes or sections that simply list relevant phrases. Third, and most appropriate, would be to include these phrases in the dictionary as entries in their own right. Each ethnopragmatic composition then can be linked to the phrases, and the meanings and subtle differences between the phrases can then be elaborated on within the dictionary proper.

\subsubsection{Roleplays/Practice}

The third key attribute of good teaching materials was the opportunity to connect knowledge to practice. This need was also reflected in the research with language teachers, who reiterated the need for these materials to be connected to classroom practice exercises. In the first instance, this is possible to do through the adaptation of existing cultural scripts to different perspectives (Sadow 2018). In particular, interactional versions of the compositions could be used to prompt roleplay exercises as well as other opportunities for practice. Dictionaries do not traditionally contain exercises such as these, but the expanding domains of online dictionaries allow for concrete connections to classroom practice to be built into the site directory, as well as the dictionary entries themselves.

\subsection{Structuring and Designing a Dictionary of Ethnopragmatics}

Most important for dictionaries, as discussed above, is the retrievability of information for the users. If the entries themselves cannot be found, the dictionary is practically useless. One of the main difficulties in creating a dictionary of ethnopragmatics is the structuring of the entries in relation to one another in the main body of the dictionary. It is essential to maintain the links between each entry and to keep strong associations between related concepts. In addition, alphabetical order is not necessarily the most retrievable for students because the headwords do not necessarily map to a user's intuition about the interaction. There are two ways to approach this problem. The first is by drawing on onomasiological lexicography (Trklja 2016), using pragmatic categories rather than semantic - such as 'making a request', 'refusing a request', etc. The second is through using lexical items as entry points to pragmatics. This approach would be difficult considering the range of expressions available or stemming from each pragmatic intention or cultural value. Either approach would require additional indexing and categorization.

In traditional e-lexicography, the back-end structure and organization do not impact on the user's experience of the dictionary. The user will still type in a word 
and be presented with the closest equivalent and be taken to the entry. In contrast, when creating a dictionary for ethnopragmatics, it is more difficult to determine what the search terms are for any single entry. As such, the search options and exploration options discussed in 5.1 are the only ways for users to find their query. In many cases for ethnopragmatic queries the explore options will be the most used options.

While throughout this chapter, I have discussed several options for different features to include in individual entries, the key to creating an effective dictionary in this context is to have a user interface that is as clean and minimalistic as possible. This means removing and hiding additional options until they are needed by the user, as shown in the wireframes in Figs. 9.3, 9.4 and 9.5. Wireframes are tools for digital design that help to lay out elements on a page. They do not reflect design decisions beyond the layout. The three wireframes show one way in which simple design can still include all of the features discussed throughout this chapter, while not appearing too dense for language learners.

\subsection{Conclusion}

Creating an ethnopragmatic dictionary of Australian English as an online resource for language learners in Australia is a much-needed component of improving education in invisible culture and developing intercultural competence in language students. To create such a dictionary, there are many considerations for the types of

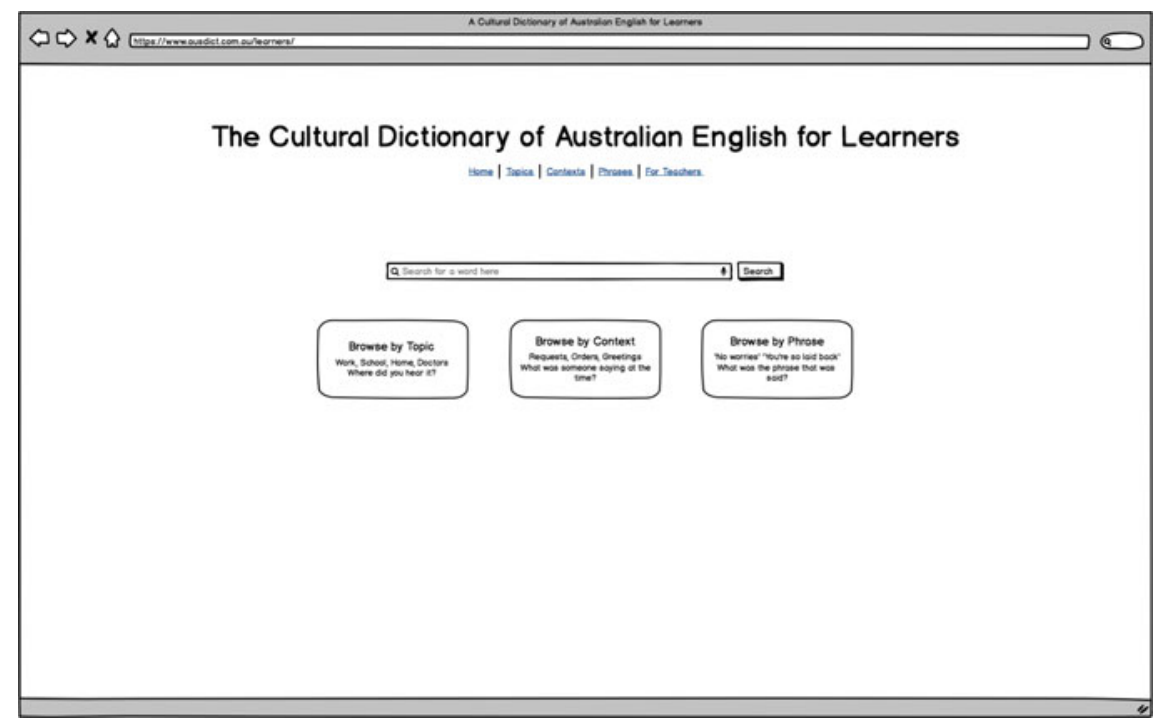

Fig. 9.3 Wireframe showing minimalist design for the proposed dictionary 


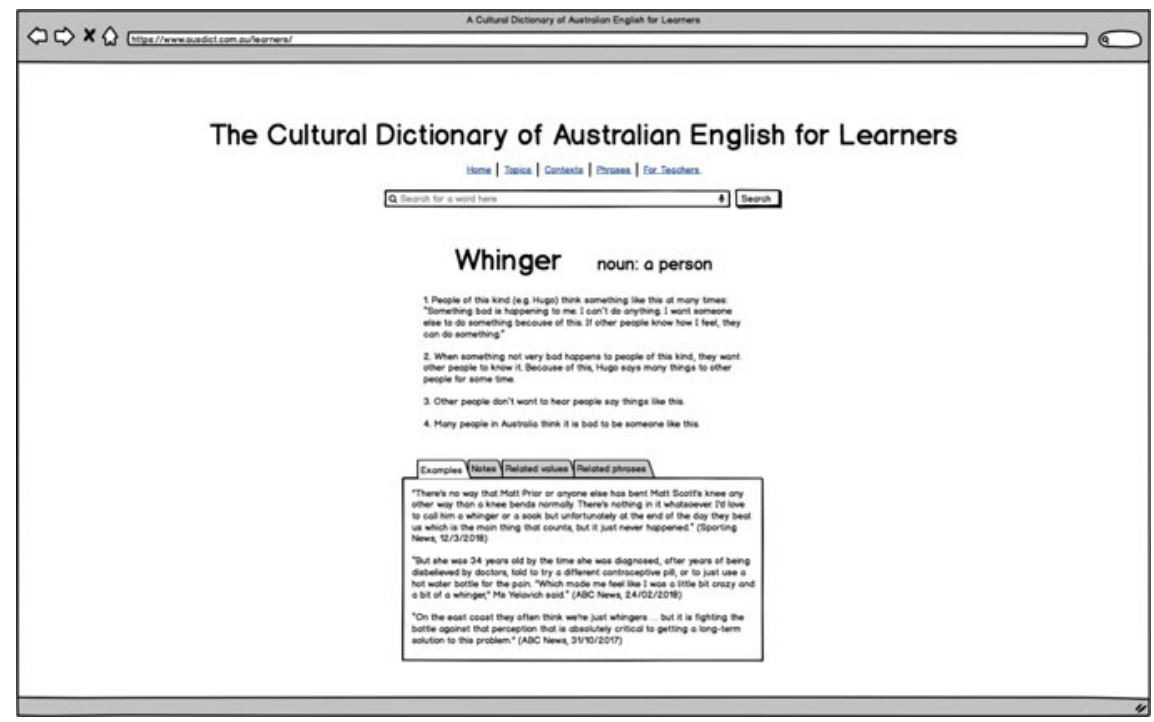

Fig. 9.4 Wireframe showing possible entry for 'whinger' from the proposed dictionary

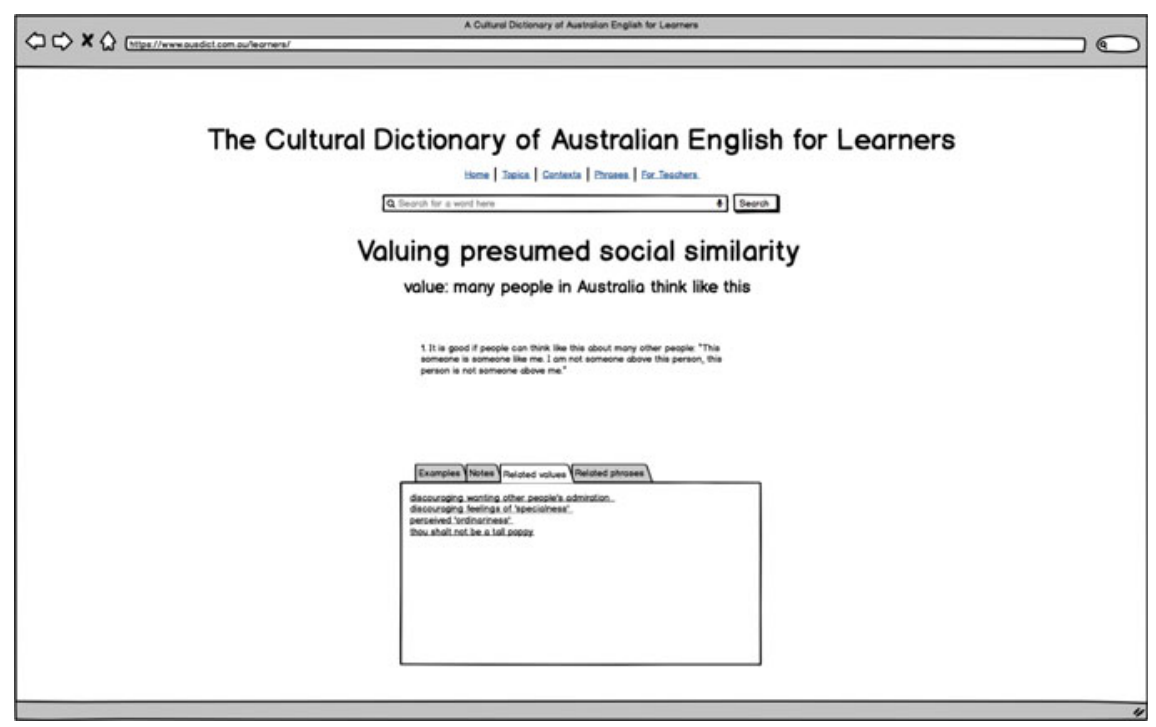

Fig. 9.5 Wireframe showing possible entry for 'valuing presumed social similarity' from the proposed dictionary 
users and the contexts in which the dictionary should be used. Most significantly, the dictionary should be aimed at decoding language use, not encoding it. This removes the pressure on language students to move towards 'native competence' and allows them to retain their own identity in language expression, while also developing their skills in interpersonal communication within a new community. Dictionaries in teaching contexts should not be viewed as exclusively dictionaries - static reference materials - but should also be seen as dynamic tools of language education, able to fulfil semantic and pragmatic functions simultaneously.

In creating an ethnopragmatic dictionary for language learners, consideration should be given to good practice for creating teaching materials within the context of intercultural language teaching. These principles encourage those materials to contain more than information, but also ways of critically engaging with that information. Using Minimal English to do this ensures that the concepts can be broken down into their components, and therefore examined and critiqued in necessary detail. The Minimal English approach also means that this can be done without valuing any one culture over another. Using this approach also creates opportunities to make explicit connections between values, attitudes and their expressions in language through similar components.

Creating these connections is not the task of Minimal English alone in such a dictionary. Including many of the traditional elements of dictionaries within a new dictionary paradigm ensures that the users can gain as much information about the language as is possible, from a number of different perspectives. At the same time, they are able to draw on familiar features to orient themselves throughout the search. Above all additional features, however, is the need for good interface design. Easy navigation and options for accessing information in the dictionary are essential to its success.

The next stages of this dictionary project are to continue with user engagement and user surveys - particularly with language learners - to determine how successful the dictionary is in actual usage, and then to adjust and update it as required. This will be undertaken in an iterative sequence as the dictionary is developed, culminating in testing of the resource in a classroom context. Following this, the dictionary will be expanded on to cover a wider range of topics and nuances in interactions.

The principles discussed in this chapter can also be applied to any NSM-based lexicographical undertaking, whether semantic or pragmatic or a combination of the two. The advantage of NSM and Minimal English is that for the first time, it provides lexicographers with a consistent descriptive methodology to capture semantic and pragmatic information, even if it is highly culturally specific. This allows lexicographers and descriptive linguists to capture the cultural components of languages - which have been missing from standard dictionaries — globally, for language learners, for migrants, and for communities speaking endangered languages.

Acknowledgements The author would like to thank Zhengdao Ye and two anonymous reviewers for their helpful comments, and Bert Peeters for his editorial assistance. 


\section{References}

ACARA. (2011). English as an additional language or dialect teacher resource. Sydney: Australian Curriculum Assessment and Reporting Authority.

Amiel, T., \& Reeves, T. C. (2008). Design-based research and educational technology: Rethinking technology and the research agenda. Journal of Educational Technology \& Society, 11(4), $29-40$.

Atkins, S., \& Rundell, M. (2008). The Oxford guide to practical lexicography. New York: Oxford University Press.

Bullock, D. (2011). NSM + LDOCE: A non-circular dictionary of English. International Journal of Lexicography, 24(2), 226-240. https://doi.org/10.1093/ijl/ecq035.

Byram, M. (1997). Teaching and assessing intercultural communicative competence. Bristol: Multilingual Matters.

Cohen, A. D., \& Ishihara, N. (2012). Pragmatics. In B. Tomlinson (Ed.), Applied linguistics and materials development. London: Bloomsbury.

Department of Education and Training. (2011). AMEP home tutor resources. Canberra: Australian Government Department of Education and Training.

Department of Social Services. (2014). Getting settled: Women refugees in Australia. Canberra: Australian Government Department of Social Services.

Fernández, S. S. (2016). Possible contributions of ethnopragmatics to second language learning and teaching. In S. Vikner, H. Jørgensen, \& E. van Gelderen (Eds.), Let us have articles betwixt us: Papers in historical and comparative linguistics in honour of Johanna L. Wood (pp. 185206). Aarhus: Aarhus University.

Fuertes-Olivera, P. A. (Ed.). (2010). Specialised dictionaries for learners. Berlin: Mouton de Gruyter. https://doi.org/10.1515/9783110231335.

Goddard, C. (2004). "Cultural scripts": A new medium for ethnopragmatic instruction. In M. Achard, \& S. Niemeier (Eds.), Cognitive linguistics, second language acquisition, and foreign language teaching (pp. 143-163). Berlin: Mouton de Gruyter. https://doi.org/10.1515/ 9783110199857.143.

Goddard, C. (2010). Cultural scripts: Applications to language teaching and intercultural communication. Studies in Pragmatics (Journal of the China Pragmatics Association), 3, 105119.

Goddard, C. (2011). Semantic analysis: A practical introduction (2nd ed.). Oxford: Oxford University Press.

Goddard, C. (2017a). Ethnopragmatic perspectives on conversational humour, with special reference to Australian English. Language \& Communication, 55, 55-68. https://doi.org/10. 1016/j.langcom.2016.09.008.

Goddard, C. (2017b). Natural semantic metalanguage and lexicography. In P. Hanks, \& G.-M. de Schryver (Eds.), International handbook of modern lexis and lexicography. Berlin: Springer. http://doi.org/10.1007/978-3-642-45369-4_14-1.

Goddard, Cliff (Ed.). (2018). Minimal English for a global world: Improved communication using fewer words. Cham: Palgrave Macmillan. https://doi.org/10.1007/978-3-319-62512-6.

Goddard, C., \& Wierzbicka, A. (2004). Cultural scripts: What are they and what are they good for. Intercultural Pragmatics, 1(2), 153-166. https://doi.org/10.1515/iprg.2004.1.2.153.

Goddard, C. (2012). Early interactions in Australian english, American english, and english english: Cultural differences and cultural scripts. Journal of Pragmatics, 44(9), 1038-1050. http://doi.org/10.1016/j.pragma.2012.04.010

Goddard, C., \& Ye, Z. (2015). Ethnopragmatics. In F. Sharifian (Ed.), The Routledge handbook of language and culture (pp. 66-83). Routledge.

Huang, C. (2016, 1 September). International students in Australia struggle on many fronts. https://www.smh.com.au/opinion/international-students-in-australia-struggle-on-many-fronts20160901-gr66dx.html. Accessed 11 September 2018. 
Kramsch, C. (1993). Context and culture in language teaching. Oxford: Oxford University Press.

Kramsch, C. (2014). Teaching foreign languages in an era of globalization: Introduction. The Modern Language Journal, 98(1), 296-311. https://doi.org/10.1111/j.1540-4781.2014.12057.x.

Landau, S. (2001). Dictionaries (2nd edn.). Cambridge: Cambridge University Press.

Levisen, C., \& Waters, S. (2017). Cultural keywords in discourse. Amsterdam: John Benjamins. https://doi.org/10.1075/pbns.277.

Lew, R., \& de Schryver, G.-M. (2014). Dictionary users in the digital revolution. International Journal of Lexicography, 27(4), 341-359. https://doi.org/10.1093/ijl/ecu011.

Liddicoat, A. (1997). Everyday speech as culture: Implications for language teaching. Australian Review of Applied Linguistics, Series S, 14, 55-70. https://doi.org/10.1075/aralss.14.03lid.

Liddicoat, A., \& Scarino, A. (2013). Intercultural language teaching and learning. West Sussex: Wiley-Blackwell. https://doi.org/10.1002/9781118482070.

Mills, N. (2018, May 1). International students say challenges in Australia include loneliness, racism, poverty. http://www.abc.net.au/news/2018-05-01/challenges-international-studentsface-in-melbourne-australia/9702606. Accessed 11 September 2018.

Moore, B. (2008). Speaking our language. Oxford: Oxford University Press.

Ostermann, C. (2015). Cognitive lexicography: A new approach to lexicography making use of cognitive semantics. Berlin: de Gruyter.

Pearson Education, Inc. (2006). Longman Essential Activator. Boston: Allyn \& Bacon.

Peeters, B. (2000). Setting the scene: Recent milestones in the lexicon-encyclopedia debate. In B. Peeters (Ed.), The lexicon-encyclopedia interface (pp. 1-52). Oxford: Elsevier Science.

Pulverness, A., \& Tomlinson, B. (2003). Materials for cultural awareness. In B. Tomlinson (Ed.), Developing materials for language teaching (pp. 443-460). London: Continuum.

Risager, K. (2006). Language and culture. Bristol: Multilingual Matters.

Sadow, L. (2018). Can cultural scripts be used for teaching interactional norms? Australian Review of Applied Linguistics, 41(1), 92-117. https://doi.org/10.1075/aral.17030.sad.

Sadow, L. (2019). An NSM-based cultural dictionary of Australian English: From theory to practice. Ph.D. thesis, Australian National University. https://doi.org/10.25911/5d514809 $475 \mathrm{cb}$

Sánchez, A. (2010). The treatment of cultural and/or encyclopaedic items in specialised dictionaries for learners. In P.A. Fuertes-Olivera (Ed.), Specialised dictionaries for learners (pp. 107-131). Berlin: Mouton de Gruyter. https://doi.org/10.1515/9783110231335.2.107.

Seargeant, P. (2011). Lexicography as a philosophy of language. Language Sciences, 33(1), 1-10. https://doi.org/10.1016/j.langsci.2010.06.002.

Tarp, S. (2008). Lexicography in the borderland between knowledge and non-knowledge. Berlin: Mouton De Gruyter.

Tomlinson, B. (2012). Materials development for language learning and teaching. Language Teaching, 45(2), 143-179. https://doi.org/10.1017/S0261444811000528.

Trklja, A. (2016). Corresponding lexical domains: A new resource for onomasiological bilingual dictionaries. International Journal of Lexicography, 36(3), ecw019-38. http://doi.org/10.1093/ ijl/ecw019.

Usó-Juan, E. (2008). The presentation and practice of the communicative act of requesting in textbooks: Focusing on modifiers. In E. A. Soler, \& M. P. Safont Jordà (Eds.), Intercultural language use and language learning (pp. 223-243). Dordrecht: Springer. http://doi.org/10. 1007/978-1-4020-5639-0_12.

Weninger, C., \& Kiss, T. (2013). Culture in EFL textbooks: A semiotic approach. TESOL Quarterly, 47(4), 694-716.

Wierzbicka, A. (1997). Understanding cultures through their key words: English, Russian, Polish, German, Japanese. New York: Oxford University Press.

Wierzbicka, A. (2002). Australian cultural scripts-bloody revisited. Journal of Pragmatics, 34 (9), 1167-1209. https://doi.org/10.1016/S0378-2166(01)00023-6.

Wierzbicka, A. (2010). Experience, evidence, and sense. Oxford: Oxford University Press. 
Wierzbicka, A. (2012). When cultural scripts clash: Miscommunication in "multicultural" Australia. In Barbara Kryk-Kastovsky (Ed.), Intercultural miscommunication past and present (pp. 121-148). Lódz: Peter Lang.

Zgusta, L. (1971). Manual of lexicography. Berlin: Walter de Gruyter. https://doi.org/10.1515/ 9783111349183.

Lauren Sadow is Sessional Academic at The Australian National University, Canberra. Her main research interests are teaching culture, interactional norms, cultural lexicography, and cross-cultural communication. Her Ph.D. thesis created an NSM-based dictionary titled The Australian Dictionary of Invisible Culture for Teachers. 